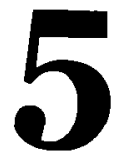

\title{
"EL PERFIL DEL PROFESOR UNIVERSITARIO DE CALIDAD DESDE LA PERSPECTIVA DEL ALUMNADO"
}

\section{('THE QUALIFIED UNIVERSITY TEACHER FROM THE STUDENTS' PERCEPTION")}

\author{
$M^{a}$ del Mar Martínez García, Begoña García Domingo y \\ José Quintanal Díaz \\ Centro de Enseñanza Superior "Don Bosco" \\ (adscrito a la Universidad Complutense de Madrid)
}

\section{RESUMEN}

En estos momentos la universidad española se encuentra inmersa en un proceso de cambio que viene marcado, por un lado, por la renovación que impone la legislación, y por otro, por unos Planes de Convergencia con Europa que parecen imponer criterios de racionalidad y eficacia a su misión. La realidad de este cambio pasará indefectiblemente por el crisol de la calidad, lo que determina que éste sea un criterio de pleno derecho a integrar en la vida académica del futuro.

Sin duda, un elemento indispensable para garantizar este proceso es la calidad docente. Pero la labor docente ha sido estudiada fundamentalmente desde la propia perspectiva del profesorado, necesitándose imprimir también el enfoque propio del destinatario. Ese es precisamente el objetivo de nuestro estudio, en el que afrontamos un diseño centrado en la opinión que el alumnado ofrece sobre las características que demanda de sus profesores.

\section{ABSTRACT}

Nowadays, Spanish University is involved in a change process due to two main reasons: firstly, the new legislation and secondly, the future plans for the European educational convergence.

From both perspectives, the quality is imposed as criteria of University efficiency. Therefore, many studies have been performed to prove that quality is a valuable criterion in the educational process; however, the teacher's role has not been analysed properly by the pupils.

Therefore, the target of this project is to consider the students' opinion about the ideal characteristics that their teachers should have. 


\section{INTRODUCCIÓN}

Todos sabemos que el concepto de calidad ha aterrizado con fuerza durante los últimos años en el campo educativo procedente del mundo de la empresa. Esta tendencia se ha manifestado en la escuela (LOE, 2006) pero también se ha hecho extensiva al ámbito universitario; la preocupación por la calidad de la enseñanza superior, en cuanto al adecuado desarrollo de sus fines y procesos, se relaciona de forma muy especial con la tarea docente e investigadora que la legislación vigente atribuye a la institución universitaria (LOU, 2001).

Tal y como señala Salvador (2005), una de las opciones a la hora de evaluar la calidad del servicio universitario se basa en la satisfacción de las expectativas de los usuarios. El aspecto básico en el que se apoya este criterio es el convencimiento de que se alcanzarán elevados niveles de calidad si se cubren las expectativas de los usuarios; es decir, su interés se centra en los criterios subjetivos de los clientes, los cuales proporcionan información acerca de las características o atributos que emplean los usuarios para evaluar la calidad. Desde este enfoque, el objetivo consistiría en adaptar el servicio a las necesidades y demandas de los clientes, lo que nos llevaría a tener que aportar los recursos y capacidades disponibles en la institución universitaria para alcanzar dicho fin.

Por otro lado, puede afirmarse que la meta primordial de la enseñanza universitaria es, a grandes rasgos, la consecución de la óptima preparación de los profesionales del futuro. Siendo esto así, resulta evidente que para conseguir el logro de tal objetivo, un factor clave será la calidad docente: sin profesores competentes la universidad no podría conseguir sus metas al no poder transmitir -o mejor dicho, no poder ayudar a construir- de forma adecuada el conocimiento. Por lo tanto, debemos considerar que el profesor ocupa un papel fundamental como referente central de todos los procesos educativos que tienen lugar en el aula y fuera de ella. No obstante, y a pesar de que en este trabajo hemos optado por centrarnos en dicho aspecto, somos conscientes de que la calidad de la enseñanza trasciende a la actuación de los profesores. Tal y como defiende Zabalza (2003) la actuación psicopedagógica de los docentes se produce en lo que él denomina un "marco de condiciones", constituido por aquellos elementos o circunstancias sobre los que los profesores no tienen capacidad de actuación y a los que deben supeditar y adecuar las decisiones que adoptan y las acciones a desarrollar. Como sostiene dicho autor, no podemos modificar la inteligencia o la preparación anterior de nuestros alumnos, ni tampoco podemos alterar el tamaño de los grupos ni cuestiones como la asignación de créditos a las materias, o la organización de espacios o recursos disponibles. Sin embargo, y he ahí un aspecto clave, sí que parte de lo que se puede considerar competencia profesional tiene que ver justamente con el hecho de ser capaces de seleccionar y llevar a cabo las actuaciones que se adapten de forma más adecuada a ese marco de condiciones que están presentes en nuestro trabajo. 


\subsection{CARACTERISTICAS QUE DETERMINAN UNA BUENA PRÁCTICA DOCENTE}

En realidad, hasta no hace demasiado tiempo, los problemas de la enseñanza universitaria han permanecido en un segundo plano si los comparamos con otros niveles educativos. Tal y como plantea Rebolloso (1995), se han ignorado problemas tan importantes como la transmisión del conocimiento o la satisfacción del alumnado en la universidad. Sin embargo, con el proceso de democratización de la enseñanza y la superación del aprendizaje fundamentado en el principio de autoridad del profesor, va tomando un creciente interés el estudio y análisis acerca del porqué se producen estos problemas y de cuáles son las características que conforman un desarrollo ideal de la docencia universitaria.

En este sentido, resulta especialmente relevante el planteamiento de Zabalza (2003) sobre lo que es la docencia de calidad, cuando propone aplicar a la enseñanza universitaria el mismo concepto de "zona de desarrollo próximo" que Vigotsky propugnaba en la educación de los niños. Zabalza (2003, p. 215) subraya que "lo que la universidad y los profesores universitarios podemos dar a nuestros estudiantes es ese plus de aprendizaje y desarrollo formativo que ellos no podrían adquirir por sí solos”. Este planteamiento, lejos de restar protagonismo al alumno como podría parecer a simple vista, lo que hace es subrayar la necesidad de una adecuada interacción en el proceso de enseñanza-aprendizaje entre el profesor y el alumno para que éste pueda llegar a construir conocimiento, superando así los simples y viejos modelos transmisivos o reproductivos que han venido imperando.

Desde esta perspectiva se hace imprescindible considerar la percepción y las expectativas del protagonista históricamente más olvidado en este proceso: el estudiante (Martínez García, 2003). Por ello, en nuestro trabajo vamos a acercarnos a las características que determinan una buena práctica docente desde la perspectiva de la satisfacción de las demandas (expectativas) del alumnado.

Por otro lado, existe otro motivo que nos lleva a interesarnos por este tema de la calidad docente. Tal y como venimos planteando, el profesor se constituye en agente clave del funcionamiento de la institución universitaria, pero en realidad, y contrariamente a lo que debería suceder por toda lógica, la función docente se encuentra claramente devaluada frente a otras como la investigación o la gestión. Esto se refleja incluso en el lenguaje, puesto que se habla de "carga" docente para referirse precisamente a la parte del trabajo correspondiente a la docencia en sí. Un profesor de enseñanza superior adquiere prestigio y promociona más bien por los resultados de sus investigaciones y publicaciones y/o por los cargos desempeñados, y no precisamente por sus cualidades docentes (Rebolloso y Pozo, 200o). Consideramos que esto entraña un serio riesgo para que realmente exista una docencia de calidad en nuestras universidades, y por lo tanto defendemos la importancia y la necesidad de la evaluación docente como vía de reconocimiento de esta función, así como su valor como instrumento de retroalimentación y mejora. 


\subsection{La evaluación docente}

Para ser profesor de educación primaria o secundaria está establecida y es aceptada la exigencia de cierta formación psicopedagógica. Sin embargo esto no es así en el ámbito de la universidad, donde los procedimientos y pruebas de ingreso del profesorado tienen poco que ver con las capacidades reales que exige la docencia (Román, 200o). Tal y como plantean Rivarosa y Perales (1998), la Universidad, de este modo, entra en una gran contradicción; de forma simultánea defiende y profesa el rigor en la investigación y en sus análisis científicos, mientras que por otro lado, prima la espontaneidad en la actividad docente, realizándose ésta de forma acrítica.

La formación psicopedagógica del profesorado universitario ha sido tradicionalmente el producto de una voluntaria autoformación y de una socialización no consciente, es decir, a través de la progresiva interiorización de lo que hemos visto o vemos hacer a los buenos profesores y la evitación de lo que vemos en los no tan buenos. No olvidemos un aspecto de suma importancia: el profesor constituye un "modelo" para sus alumnos, y tiene más peso su forma de actuar, es decir, la coherencia entre lo que dice y lo que hace en cada tema concreto, que sus aportaciones teóricas; a menudo pesa más el cómo que el qué.

Como refleja Mayor (1997), los conocimientos de naturaleza psicológica y pedagógica útiles para la mejora de la docencia universitaria los va construyendo cada profesor a partir de su asistencia a congresos y otras reuniones científicas, seminarios ad hoc, lecturas personales, intercambio de experiencias con otros compañeros y, por supuesto, las reflexiones sobre la propia práctica docente. Precisamente nuestro trabajo se vincula a esta última vía de mejora, puesto que tal y como plantean Ramos y Rodríguez (2000), la comparación de las características teóricas del profesor eficaz con la propia acción docente, constituye uno de los cuatro procedimientos que puede utilizar un profesor para su autoevaluación.

Por otro lado, consideramos que la evaluación docente debe trascender el espacio concreto de las aulas y de lo que se hace en ellas. Como defiende Zabalza (2000), analizar la docencia implica tomar en consideración al menos tres momentos básicos: la preparación, el desarrollo de la intervención didáctica directa y las acciones posteriores que siguen formando parte de la enseñanza, como es la evaluación o las acciones de tutoría. Además, tal y como sostiene dicho autor, la enseñanza trasciende el ámbito de lo visible, lo objetivo y lo cuantificable; hay aspectos, que pertenecen o se derivan de los particulares puntos de vista, actitudes o sistemas de interpretación de los participantes en el proceso de enseñanza-aprendizaje.

\subsection{Objetivos}

Dada la enorme relevancia de la figura del docente, nos preocupa e interesa conocer cuáles han de ser las características que lo definan para elaborar un perfil del profesor universitario de calidad. 
No obstante, la revisión pormenorizada de estudios similares desarrollados por otros autores ponen claramente de manifiesto que sería muy difícil alcanzar un perfil único, ya que éste dependerá de multitud de variables y condicionantes, entre los que cabría destacar tanto la metodología empleada como la fuente de la que obtuviésemos la información. De este modo, algunos los autores han planteando perfiles no siempre concordantes (Ibáñez, 1990; González y Fuentes, 1997; Beltrán, 2000; Apodaca y Grad, 2002), lo cual evidencia que la diversidad de planteamientos con que podemos encontrarnos complica la pretensión de establecer un perfil único, o cuanto menos, concitar un modelo referencial más o menos uniforme de actuación profesional o relacional del docente.

Por eso, en nuestro trabajo hemos acotado el campo de estudio dedicándonos a analizar en qué medida las cualidades del profesor satisfacen las demandas (expectativas) del alumnado. En definitiva, el objetivo final que nos proponemos es conocer el perfil (características, cualidades, condición, ...) que en opinión de los alumnos universitarios deben presentar sus profesores en los distintos ámbitos de su actuación docente-pedagógica, investigadora y personal-, conocimiento que estimamos podrá servir como vehículo de reflexión y retroalimentación que favorezca el desarrollo de nuestra labor profesional.

Hemos optado por utilizar un instrumento de evaluación sensible a la detección de variables afectivas y actitudinales que están presentes en la percepción que los estudiantes tienen de la docencia universitaria. Por ello hemos elegido un cuestionario con escala tipo Likert como instrumento de medida, puesto que además, aunque la evaluación del profesorado a través de la encuesta de opinión de los estudiantes ha sido un método comúnmente utilizado, no ha sido el caso de la Escala Likert (Rebolloso, 2000).

Consideramos que con este instrumento, además de poder establecer los atributos del "profesor ideal" se abre la posibilidad de acceder a componentes evaluativos, emocionales o afectivos ligados a dicho concepto por parte de los alumnos, lo cual, tal y como venimos defendiendo, creemos esencial para mejorar los procesos de enseñanza-aprendizaje en el seno de la Universidad.

Así pues, partimos de los siguientes presupuestos:

a. El docente universitario, debería responder a un perfil claramente definido, determinado por la meta de alcanzar una acción educadora basada en la calidad y la eficacia.

b. El concepto que de la calidad docente tienen los alumnos universitarios debería servirle al profesor para:

- Optimizar los procesos de enseñanza y someterlos a dicha exigencia.

- Optar por un estilo concreto de relación, intervención e implicación en la acción educativa. 
De forma más concreta, nuestra investigación responderá a los siguientes objetivos específicos:

1. Identificar las características determinantes del criterio de calidad en el perfil del docente universitario desde el punto de vista del alumno.

2. Construir el instrumento de medida que nos permita determinar el valor otorgado por el alumnado universitario a las cualidades referidas en el objetivo anterior.

\section{METODOLOGÍA}

Al objeto de conocer cuáles deben ser las cualidades que caractericen a un profesor universitario "de calidad" desde el punto de vista de los alumnos, se plantea recoger su opinión mediante un procedimiento de ENCUESTA en dos fases sucesivas:

1. Fase I: Elaboración y codificación del instrumento inicial de recogida de datos (cuestionario de preguntas abiertas).

2. Fase II: Elaboración y aplicación de un Cuestionario con escala de tipo Likert.

A continuación pasamos a describir tanto la muestra como el procedimiento planteado.

\subsection{Muestra}

Para la elaboración y codificación del instrumento inicial de recogida de datos se trabaja con una muestra de 320 estudiantes de distintas universidades, habiendo sido éstos seleccionados con una técnica de muestreo no probabilístico (muestreo incidental, invitado o 2 a propósito"):

- Universidad de Alcalá de Henares: 43 sujetos.

- Universidad Nacional de Educación a Distancia: 15 sujetos.

- Universidad Complutense de Madrid: 47 sujetos.

- Universidad de Salamanca: 92 sujetos.

- Centro de Enseñanza Superior "Don Bosco" de Madrid: 123 sujetos.
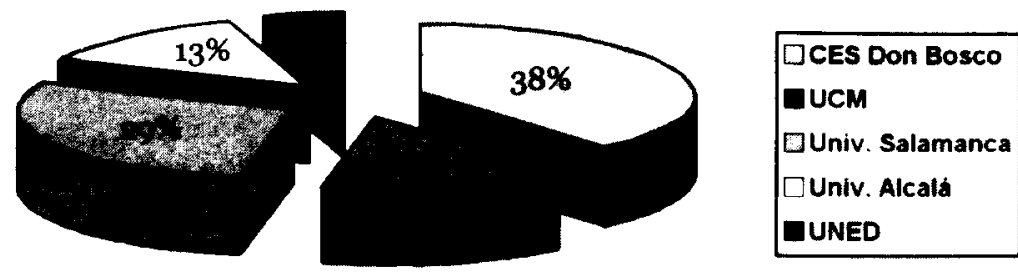

Gráfico 1: representación por sectores de la procedencia de la muestra. 


\subsection{Procedimiento}

Aplicando un cuestionario de preguntas abiertas (ver Anexo) se obtuvieron los calificativos que más frecuentemente se consideran característicos de un buen profesor universitario en dos ámbitos diferentes: CÓMO ES (físicamente, personalmente y en el ámbito relacional) y CÓMO HACE SU TRABAJO (labor docente, relaciones con alumnos y otras actividades que debe desarrollar). Debemos decir que de forma previa, la selección de las facetas incluidas en dicho cuestionario de preguntas abiertas fue determinada a partir de un pretest, mediante el cual se reformuló un protocolo de prueba en base a las sugerencias y críticas de una pequeña muestra de alumnos universitarios invitados. En este primer protocolo se incluían los siguientes aspectos: CÓMO ES (apariencia física, carácter/personalidad y como profesional), COMO HACE SU TRABAJO (actividad docente y ámbito relacional) y OTROS, que finalmente quedaron reformulados del modo mencionado arriba, de manera que el apartado de CÓMO ES COMO PROFESIONAL desapareció para quedar incluido en el CÓMO HACE SU TRABAJO apareciendo en su lugar el apartado en el que pretendíamos que quedara reflejado CÓMO ES EN SUS RELACIONES INTERPERSONALES. Por último, y ante las sugerencias recogidas a partir de este pretest, decidimos incluir un aspecto que complementara la labor del docente como profesional más allá de la actividad propia del aula, al que titulamos OTRAS TAREAS O ACTIVIDADES QUE CONSIDERAS DEBE REALIZAR.

Esta primera encuesta, de modalidad autoadministrada y transversal, se cumplimentó entre el 13 de abril y el 14 de mayo de 2005, siendo aplicada, tal y como ya hemos señalado, a una muestra de 320 estudiantes universitarios de distintos centros.

\subsection{Codificación}

Una vez recogida esta información, se procedió a la codificación de la misma siguiendo el procedimiento que describimos a continuación. Para cada uno de los siete ámbitos considerados como potencialmente descriptivos de un profesor universitario y que han sido mencionados al principio de este apartado, se elaboró un listado que recogía todos los adjetivos empleados por los alumnos así como su frecuencia absoluta de aparición, siendo necesario, en muchas ocasiones, transformar los comentarios literales de los alumnos en el calificativo que mejor representara su idea inicial'.

\section{RESULTADOS}

Finalizado este proceso, el número total de calificativos relativos a cada uno de los ámbitos aparece recogido en la TABLA 1. 


\section{CÓMO ES}

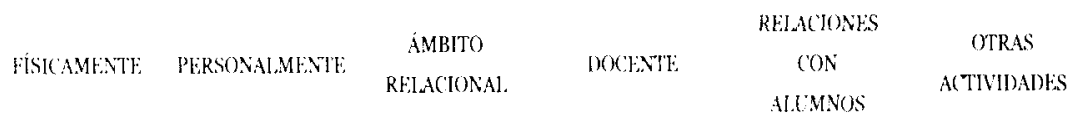

\section{CÓMO HACE SU TRABAJO OTROS}

$\begin{array}{lllllll}30 & 114 & 70 & 61 & 90 & 44 & 15\end{array}$

$\begin{array}{lllllll}30 & 114 & 70 & 61 & 90 & 44 & 15\end{array}$

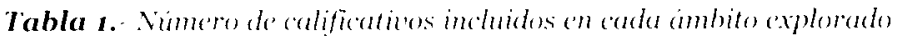

Como era de esperar, dentro de cada una de las categorías deseritas aparecía gran cantidad de adjetivos que hacian referencia a la misma cualidad, por lo que procedimos a aglutinarlos en función de su significado empleando para ello el Diccionario de Sinónimos de la Real Academia de la Lengua Española.

\begin{tabular}{|c|c|c|c|c|}
\hline Afeitado & Buena apariencia & Acogedor & Buena presencia & Modelo para alumnos \\
\hline Aseado & Diferenciado & $\begin{array}{l}\text { Coherente con su } \\
\text { edad }\end{array}$ & Estilo personal & No viejo \\
\hline Correcto & No importa & Bien vestido & Actual & Buena forma \\
\hline Formal & Saludable & Neutro & Cuidado & Corrección posturial \\
\hline Imponga respeto & Arreglado & Adecuado al contexto & Cómodo & Joven \\
\hline Serio & Indiferente & Normal & Moderno & Atractivo \\
\hline
\end{tabular}

Tabla 2. (')Mo lis: IFSK AMENT:

$\begin{array}{lllll}\text { Captador de atención } & \text { Entusiasta } & \text { No pedante } & \text { No rencoroso } & \text { Reflexivo } \\ \text { Abierto } & \text { Coherente } & \text { Democrático } & \begin{array}{l}\text { Inquieto } \\ \text { intelectualmente } \\ \text { Independiente }\end{array} & \text { Objetivo } \\ \text { Accesible } & \text { Crítico } & \text { Empático } & \text { Ordenado } \\ \text { Activo } & \text { Comunicativo } & \text { Seguro } & \begin{array}{l}\text { Preocupado } \\ \text { por sus alumnos }\end{array} \\ \text { Adaptativo } & \text { Cariñoso } & \text { Flexible } & \text { Motivador } & \text { Participativo } \\ \text { Agradable } & \text { Culto } & \text { Extrovertido } & \text { Ecuánime } & \text { Puntual } \\ \text { Amable } & \text { Cooperativo } & \text { Educado } & \text { Íntegro } & \text { Observador } \\ \text { Ambicioso } & \text { Conciliador } & \text { Simpático } & \text { Liberal } & \text { Práctico } \\ \text { Apasionado } & \text { Confia en los alumnos } & \text { Firme } & \text { Divertido } & \text { Prudente } \\ \text { Asertivo } & \text { Comunicador } & \text { Espontáneo } & \text { Jovial } & \text { Fuerte } \\ \text { Astuto } & \text { Competente } & \text { Afirmado } & \text { Alegre } & \text { Profesional } \\ \text { Atento } & \text { Cordial } & \text { Eficaz } & \text { Inteligente } & \text { Paciente }\end{array}$




$\begin{array}{lllll}\text { Autoridad } & \text { Correcto } & \text { Exigente } & \text { Innovador } & \text { Persistente } \\ \text { Cercano } & \text { Disponible } & \text { Ideas claras } & \text { Natural } & \text { Razonable } \\ \text { Comprensivo } & \text { Dinámico } & \text { Humilde } & \text { Carismático } & \text { Realista } \\ \text { Comprometido } & \text { No aburrido } & \text { Honesto } & \text { No paternalista } & \text { Responsable } \\ \text { Constante } & \text { Disciplinado } & \text { Implicado } & \text { Optimista } & \text { Sincero } \\ \text { Controlado } & \text { Compañerismo } & \text { Saber estar } & \text { Motivado } & \text { Solidario } \\ \text { Creativo } & \text { Dialogante } & \text { Interesante } & \text { No racista } & \text { Serio } \\ \text { Equilibrado } & \text { Carismático } & \text { Humano } & \text { Notoriedad } & \text { Respetuoso } \\ \text { Estable } & \text { Convincente } & \text { Formal } & \text { Maduro } & \text { Receptivo } \\ \text { Sentido del humor } & \text { Sensato } & \text { Sereno } & \text { Trabajador } & \\ \text { Soñador } & \text { Sencillo } & \text { Sociable } & \text { Tolerante } & \text { Tranquilo }\end{array}$

Tabla 3.- (OMO KS: PERSONALMFNTE

$\begin{array}{lllll}\text { Abierto } & \text { Creativo } & \text { Justo } & \text { Exigente } & \text { Simpático } \\ \text { Accesible } & \text { Disponible } & \text { Mediador } & \text { Flexible } & \text { Sincero } \\ \text { Afable } & \text { Desinteresado } & \text { Inspirador } & \text { Implicado } & \text { Claro } \\ \text { Afectuoso } & \text { Confidente } & \text { Jovial } & \text { Equitativo } & \text { Sociable } \\ \text { Agradable } & \text { Dinámico } & \text { Motivador } & \text { Fiel } & \text { Sensible } \\ \text { Amable } & \text { Constante } & \text { Interesante } & \text { No racista } & \text { Serio } \\ \text { Ameno } & \text { Educado } & \text { Humano } & \text { Notoriedad } & \text { Respetuoso } \\ \text { Amigable } & \text { Crítico } & \text { Formal } & \text { Maduro } & \text { Receptivo } \\ \text { Asertivo } & \text { Diplomático } & \text { Sereno } & \text { Trabajador } & \text { Cercano } \\ \text { Atento } & \text { Coherente } & \text { Sociable } & \text { Tolerante } & \text { Tranquilo } \\ \text { Autocrítico } & \text { Discreto } & \text { Sereno } & \text { Trabajador } & \text { Correcto } \\ \text { Autoridad } & \text { Dialogante } & \text { Libre } & \text { Entrañable } & \text { Sencillo } \\ \text { Buen compañero } & \text { Empático } & \text { Paciente } & \text { Comprensivo } & \text { Comunicador } \\ \text { Buena persona } & \text { En su sitio } & \text { Preocupado por los } & \text { Cercano } & \text { Correcto }\end{array}$

Tabla 4. (OOMO ES: AMBITO RELACIONAI. 


\begin{tabular}{|c|c|c|c|c|}
\hline Abierto & Crítico & Formado & Motivado & Receptivo \\
\hline Artive & Disponible & Actualizado & Orgamiato & Com recursos \\
\hline Ajustado al programa & Concreto & Empático & Interactivo & Puntual \\
\hline Anleno & Congruente & Espontanero & Justo & Mectiador \\
\hline Analítico & Dinámico & Flexible & Orientado a la práctica & Reflexivo \\
\hline$M(n+n)$ & Constante & (ooperativo & Moderador & Responsithle \\
\hline Autoevaluador & Divertido & Facilitador & Orientador & Serio \\
\hline Auturidad & Coluborador & Entusiasta & Hotiador & Respertuoso \\
\hline Ayuda en tutorias & Coherente & Exigente & Libertad de expesión & Repite explicaciones \\
\hline Clars & Eficiz & Imonater & Pinciente & Vincicional \\
\hline Comunicativo & Disciplinado & Interesante & Promueve participación & Tranquilo \\
\hline Cimpiliabr & Dorennentado & Implicutedo & Participatius & Siguren \\
\hline
\end{tabular}

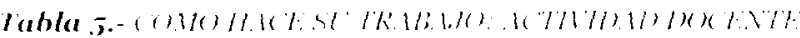

\begin{tabular}{|c|c|c|c|c|}
\hline Abierto & Comprometido & Disciplinado & Interactivo & Receptivo \\
\hline Aremilhe & (ereann & Enpratios & Intemsidad & Simern \\
\hline Activo & Claro & Enriquecedor & Objetivo & Tolerante \\
\hline 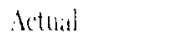 & Interaction & Butrovertido & Relacisin frecuente & Semoro \\
\hline Afectuoso & Con autoridad & Educado & Llega al alumno & Servicial \\
\hline Agradable & Companerismo? & Denocration & Igualitarios & Resiolution \\
\hline Ameno & Crítico & Estable & Libertad & Serio \\
\hline Imistoso & ('omunicativo & Silte escuchall & histot & Simpriticu \\
\hline Animador & Culto & Estimulador & Natural & Tradicional \\
\hline Atentr) & ('reativo & Exigente & Motivador & Tintor \\
\hline Buena relación & Disponible & Relación fluida & Ordenado & Preocupado \\
\hline Cercano & Discreto & Humatno & Con persomalidad & Colaborador \\
\hline Complicidad & Didáctico & Familiar & Participativo & Responsable \\
\hline comprension & Dialogante & (oluerentes & Dinamico & Dedicade \\
\hline
\end{tabular}




\begin{tabular}{|c|c|c|c|c|}
\hline Confinaza & Wistendido & Praxe & Noledont & Wonets \\
\hline Conocimiento & Distante & Frío & Paciente & Sentido del humor \\
\hline Cooperestivo & Thellitudor & fertlle & 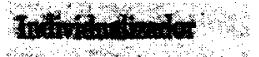 & trates \\
\hline Cordial & Directo & Generoso & Positivo & Implicado \\
\hline
\end{tabular}

Tabla 6.- CÓMO HACE SU TRABAJO: RELACIÓN CON LOS ALUMNOS

\begin{tabular}{|c|c|c|c|c|}
\hline Ayodi a los ahunnos & Buen componieno & Dilogante & thands & outument \\
\hline Aporta materiales & Trato individualizado & Dinámico & Formación continua & $\begin{array}{l}\text { Actividades fuera } \\
\text { del aula }\end{array}$ \\
\hline Acososible & $\begin{array}{l}\text { Preacupine por ov } \\
\text { alumane }\end{array}$ & 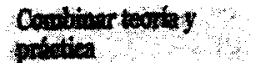 & 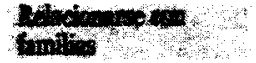 & A \\
\hline Actualizarse & Cualificado & Evaluación conjunta & $\begin{array}{l}\text { Implicarse problemas } \\
\text { alumnos }\end{array}$ & $\begin{array}{l}\text { Evaluación continua de } \\
\text { adquisición conocimientos }\end{array}$ \\
\hline Activo & Cooglinenc ow otue & 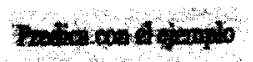 & Dencents & $+1+28+4=$ \\
\hline Ajustado al grupo & Culto & Con sentido del humor & Investiga & $\begin{array}{l}\text { Satisfecho con su } \\
\text { profesión }\end{array}$ \\
\hline Autoes & Com & & tim & Wrate \\
\hline Reciclarse & Lenguaje comprensible & $\begin{array}{l}\text { Innovador en materiales } \\
\text { y actividades }\end{array}$ & Orien & \\
\hline
\end{tabular}

Tabla 7.- CÓMO HACE SU TRABAJO: OTRAS TAREAS A REALIZAR

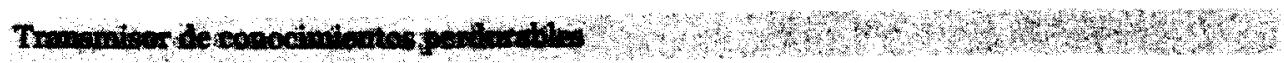

Más práctica y menos teoría inútil

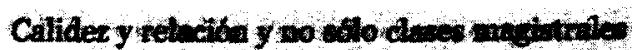

Que enseñe desde la experiencia

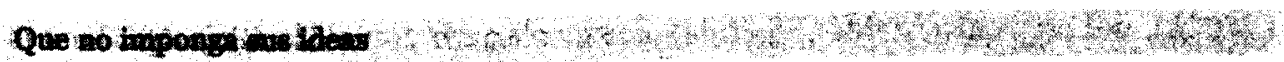

Buena persona: humilde y abierto a enriquecerse

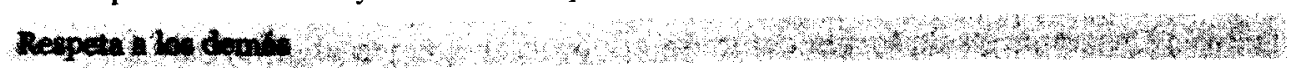
Cumple con sus obligaciones

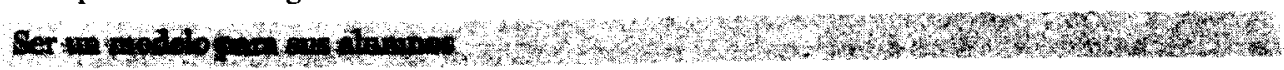

Dar libertad a los alumnos como adultos

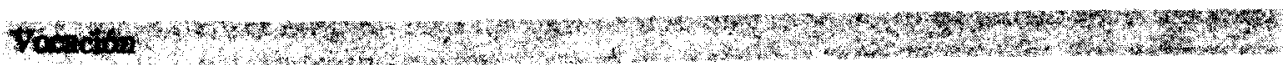

Organiza cursos o seminarios

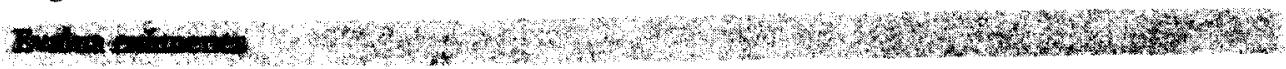

Jubilarse a tiempo: con ello se evita quedar anticuado metodológicamente y perder la motivación

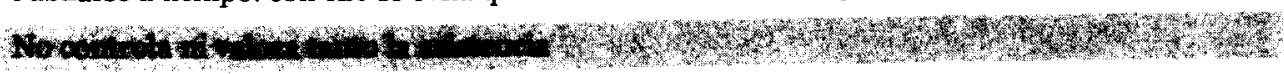

Tabla 8.- OTROS (15 “citas literales" expresadas por los encuestados) 
Tras este proceso, los 433 adjetivos codificados inicialmente quedaron reducidos a un total de 47 , distribuidos según se recoge en la tabla 9.

\section{COMO RS}

APARIENCIA FÍSICA

Limpio Arreglado Saludable Atractivo Joven Moderno Natural

\section{CARACTERÍSTICAS PERSONALES}

Equilibrado
Inteligente
Humano
Estable
Educado
Seguro
Responsable
Flexible
Puntual
Humilde

COMPETENCIA PROFESIONAL

OTros

\section{SOCIALES}

Amable
Cordial
Abierto
Simpático
Implicado
Respetuoso
Integrado

Actualizado Investigador Facilitador Orientador

Motivado
Formado
Cercano
Firme
Crítico-reflexivo
Coherente
Justo
Dinámico
Motivador
Comunicador
Ameno
Disciplinado
Claro
Eficaz
Exigente
Competente
Práctico
Directivo
Participativo

19

Tabla 9.- Calificativos incluidos en cada ámbito explorado una vez aglutinados por significados.

Como puede observarse en la tabla 9 el número final de ámbitos considerados pasó a ser de 5 frente a los 7 iniciales puesto que se decidió que el ÁMBITO RELACIONAL del apartado CÓMO ES (tabla 4) se solapaba con el de RELACIÓN CON LOS ALUMNOS (del apartado CÓMO HACE SU TRABAJO (tabla 6). Por la misma razón, los subapartados de LABOR DOCENTE (tabla 5) y OTRAS ACTIVIDADES (tabla 7) se tomaron de forma conjunta al observar que los alumnos aludían a las mismas cualidades en ellos.

Finalmente, estos adjetivos se transforman en 47 pares de antónimos (empleando de nuevo un diccionario) que nos permitirán construir el diferencial semántico (Osgood y otros, 1976), con el que determinar en qué medida las cualidades allí recogidas son relevantes para definir a un buen profesor universitario.

\section{4.- CONCLUSIONES}

La presente investigación es desarrollada bajo un marcado carácter interdepartamental, con la humilde pretensión de responder, en nuestro marco universitario, a las necesidades de evaluación y mejora de la labor docente en su actuación 
cotidiana, que resultan ineludibles en los procesos de calidad en los que actualmente nos encontramos inmersos.

En este sentido señalaremos que el marco de actuación técnica de nuestro trabajo, queda reducido a las instalaciones brindadas por el Centro de Enseñanza Superior en Humanidades y Ciencias de la Educación Don Bosco, de Madrid, a través de sus Áreas de Enseñanza de Psicología Evolutiva y de la Educación y Pedagogía, cuya instrumentación y tecnología queda a disposición del Equipo de Investigación.

Como prospectiva nos proponemos, una vez que ya tenemos construido el instrumento definitivo de recogida de datos, proceder a aplicarlo a una amplia y variada muestra de estudiantes universitarios de diferentes ciclos, especialidad académica y tipo de centro de estudios, con el objetivo de comprobar si existen diferencias significativas en el perfil del "profesor ideal" que se tiene a lo largo de los distintos cursos de formación universitaria, en diversas carreras y en diferentes modalidades de centros de estudio (privados, públicos, "a distancia”).

En este sentido queremos destacar que contaremos con la valiosísima contribución que va a suponer la Red Internet para proyectar nuestro estudio a otras Universidades fuera de Madrid, tanto nacionales como internacionales, y de este modo enriquecer el estudio y el conocimiento adquirido con una perspectiva mucho más amplia y abierta que la que nos pudiera imponer el marco universitario de la Comunidad de Madrid. Así, por un lado aprovecharemos los contactos oficialmente establecidos por nuestro Centro Universitario, a través de la Universidad Complutense, con las demás Universidades (públicas y privadas de ámbito nacional) y por otro, trabajaremos en el contexto internacional en el marco de los programas de intercambio y experiencia compartida de los Programas Sócrates de la Unión Europea o el plan transnacional que brindan las IUS (Instituciones Universitarias Salesianas) en una cincuentena de centros universitarios de los cinco continentes. Todo ello nos ofrece un marco de desarrollo lo suficientemente amplio para nuestro estudio y rico como para intentar dar respuesta a los objetivos planteados.

Respecto a Internet, señalaremos que se ha convertido en un importante y valiosísimo instrumento de trabajo, permitiéndonos presentar una encuesta en la Red y recibir cuantas aportaciones las instituciones reseñadas nos puedan ofrecer. Será la principal fuente documental, y de igual modo un medio de comunicación excelente para abrir nuestras expectativas a un contexto tan amplio como es la universidad a escala global.

Una vez alcanzados los objetivos programados tras la conclusión de nuestra segunda fase de investigación podremos presentar a la Comunidad Universitaria -y así es nuestra intención-, un perfil adecuado del profesor de calidad, determinado por la perspectiva discente del mismo, y contribuir a enriquecer en este aspecto los procesos de Análisis de Calidad que en los distintos centros de formación universitaria se están llevando a cabo. 


\section{ANEXO: Cuestionario de preguntas abiertas}

\section{LA CALIDAD DEL PROFESORADO UNIVERSITARIO}

Estamos solicitando vuestra colaboración con el objetivo de conocer la perspectiva que tenéis como alumnos/as acerca de las características que debe poseer un profesor/a universitario de "calidad"; es decir, nos interesa conocer cuáles son las cualidades que consideráis propias de un buen docente universitario. Para ello, sólo tenéis que indicarlas en cada uno de los apartados que os proponemos.

\section{MUCHAS GRACLAS POR TU PARTICIPACIÓN}

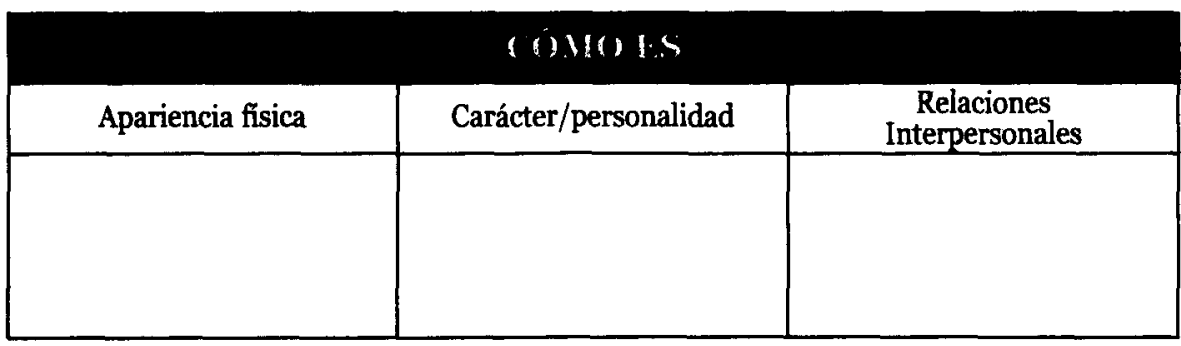

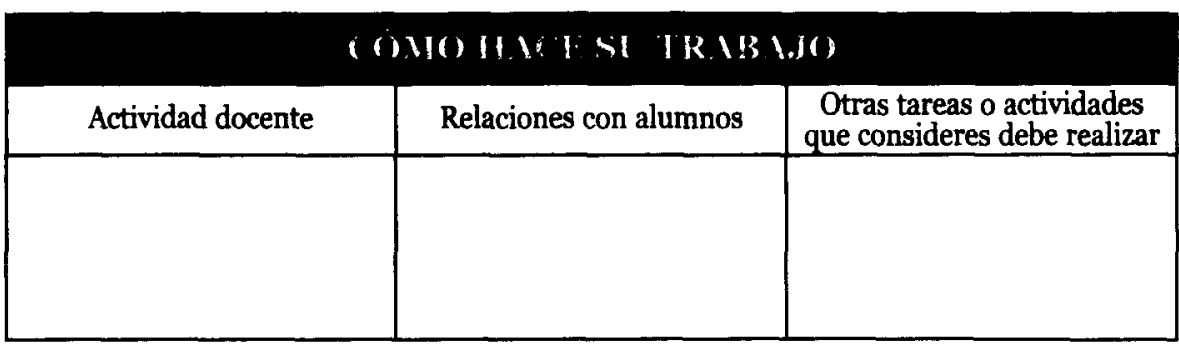

\section{() IKas}




\section{NOTAS}

1.- En orden a garantizar la fiabilidad, tanto intra como interjueces, cada uno de los miembros del equipo codificó por separado dos de los siete ámbitos explorados empleando el apartado de APARIENCIA FISICA (el menos subjetivo de todos) como oportunidad práctica para mostrar criterios unánimes de codificación a la globalidad del equipo.

\section{REFERENCLAS BIBLIOGRÁFICAS}

Apodaca, P. y Grad, H. (2002). Análisis dimensional de las opiniones de los alumnos universitarios sobre sus profesores. Revista de Investigación Educativa, 20 (2), 385-409.

Beltrán, J. (2000.) Intervención psicopedagógica y curriculo escolar. Madrid: Pirámide.

González, M. y Fuentes, E. J. (1997). El profesorado universitario: reflexiones en torno a su formación y a su desarrollo profesional. Adaxe, 13, 85-101.

Ibáñez, J. A. (1990). Dimensiones de la competencia profesional del profesor de universidad. Revista Española de Pedagogía, 186, 239-255.

Martínez García, M. M. (2003). "La mirada del educando", en Quintanal, J. (coord). Los retos de la educación en el siglo XXI. Madrid: CES Don Bosco - Edebé, 121-142.

Mayor, C. (1997). El perfeccionamiento de los profesores universitarios principiantes a juicio de sus alumnos. Revista Interuniversitaria de Formación del Profesorado, 30, 127-149.

Osgood, Ch. E. y otros (1976). La medida del significado. Madrid: Gredos.

Ramos, J. L. y Rodríguez García, L. M. (2000). Autoevaluación del profesorado: un reto para su desarrollo profesional y para la mejora de la calidad educativa en el siglo XXI. Actas del X Congreso INFAD, 856-860.
Rebolloso, E. y otros (1995). Una aproximación a la definición de "profesor ideal". Dimensiones aportadas desde la perspectiva del alumno universitario. Actas del $V$ Congreso Nacional de Psicología Social, (5), 127-141.

Rebolloso, E. y Pozo, C. (2000). Las actitudes de los estudiantes universitarios hacia sus profesores: implicaciones para la mejora de la calidad docente. Revista Psicología Educativa, 6 (1), 27-50.

Rivarosa, A. y Perales, F. J. (1998). La transformación de la pedagogía de los profesores universitarios de ciencias desde la reflexión: una propuesta innovadora. Revista Interuniversitaria de Formación del Profesorado, 33, 141- 159.

Román, J. M. (2000). Evaluación de la formación psicopedagógica de profesores universitarios. Actas del $X$ Congreso INFAD, 832-846.

Salvador, C. M. (2005). La percepción del cliente de los elementos determinantes de la calidad del servicio universitario: características del servicio y habilidades profesionales. Papeles del Psicólogo, 26 (90), 1-9.

Zabalza, M. A. (2003). Competencias docentes del profesorado universitario. Calidad y desarrollo profesional. Madrid: Narcea. 


\section{PALABRAS ClaVE}

Perfil docente, calidad educativa, enseñanza universitaria.

\section{KEY WORDS}

Teaching profile, quality of education, university education.

\section{PERFIL ACADEMICO DE LOS AUTORES}

Ma del Mar Martínez García, Licenciada en Psicología por la Universidad Pontificia de Salamanca, Máster en Psicología Clínica Infantil y Experto Universitario en Práctica Psicomotriz Educativa, profesora titular y coordinadora del Área de Enseñanza e Investigación de Psicología Evolutiva y de la Educación del Centro de Enseñanza Superior Don Bosco de Madrid (centro adscrito a la Universidad Complutense de Madrid).

Begoña García Domingo, Doctora en Psicología por la Universidad Autónoma de Madrid, profesora titular del Área de Enseñanza e Investigación de Psicología Evolutiva y de la Educación del Centro de Enseñanza Superior Don Bosco (centro adscrito a la Universidad Complutense de Madrid).

José Quintanal Díaz. Doctor en Ciencias de la Educación por la UNED, profesor titular del Área de Enseñanza e Investigación de Pedagogía del Centro de Enseñanza Superior Don Bosco (centro adscrito a la Universidad Complutense de Madrid) y Profesor Asociado de la Facultad de Educación de la UNED.

Dirección de los autores: Centro de Enseñanza Superior en Humanidades y Ciencias de la Educación "Don Bosco"

C/ María Auxiliadora, 9 28040 Madrid

E-mail: marmar@cesdonbosco.com bgd@cesdonbosco.com quintanal@cesdonbosco.com

Fecha recepción del artículo: 16. mayo. 2006 Fecha aceptación del artículo: 12. julio. 2006 